\title{
Hybrid LES - Review and assessment
}

\author{
S VENGADESAN $^{1}$ and P NITHIARASU ${ }^{2}$ \\ ${ }^{1}$ Department of Applied Mechanics, Indian Institute of Technology, \\ Chennai 600036 \\ ${ }^{2}$ Civil \& Computational Engineering Center, School of Engineering, \\ University of Wales Swansea, Swansea SA2 8PP, UK \\ e-mail: vengades@iitm.ac.in
}

MS received 7 November 2005; accepted 3 May 2007

\begin{abstract}
In the late eighties and up to the beginning of nineties computation of turbulent flows is mostly dominated by RANS (Reynolds Averaged Navier-Stokes Simulation) type modelling. During the last few years URANS (Unsteady RANS) and LES (Large Eddy Simulation) type of approaches have been attempted with some success. Yet, there have been many difficulties when LES is applied to practical engineering problems and to high Reynolds number flows as energy dissipating eddies become really small and mesh resolution required for a reasonably resolved LES approaches that of DNS (Direct Numerical Simulation). An alternative solution suggested was to combine RANS and LES, which in general referred to as Hybrid LES. There have been many proposals for combining RANS and LES in different ways. In this article, some of the issues involved in performing hybrid LES reported in the recent literature is briefly reviewed.
\end{abstract}

Keywords. DNS; Hybrid LES; RANS; interface smoothing and conditions.

\section{Introduction}

Most of the practically important flows are turbulent in nature. With the advent of everincreasing power of digital computers, the developments of accurate and efficient numerical schemes have also been on the rise. It is now possible to perform numerical calculations of turbulent flows of reasonable size engineering problems. It is well known that the NavierStokes equations can describe the unsteady turbulent flows. However, to reduce the cost of computation, often Reynolds Averaged Navier-Stokes (RANS) equations are employed. The effect of turbulent fluctuations that appear in RANS equations must be modelled to close the system. A wide variety of models is available ranging from simple, algebraic models to two equation models, to Reynolds stress closures. A comprehensive review of such turbulence models can be found in Johnson (1998), Peyret \& Krause (2000), Pope (2000) and Wilcox (2006). RANS solutions are used successfully in solving engineering applications that use fairly complex flow configurations. However, RANS approach suffers from a limitation that the model is not able to represent the effects of fluctuations over a very wide range of scales. 
Although the small-scale motions are universal, the large scales are dependent on boundary conditions and it may not be possible to accurately model the effect of large scale turbulence in different flow regimes using RANS approach. Alternative ways of computing turbulent flows are Direct Numerical Simulation (DNS) and Large Eddy simulation (LES). In this report a brief overview of these techniques and current developments in hybrid LES techniques are discussed.

\section{Direct numerical simulation (DNS)}

Direct Numerical Simulation (DNS) of the turbulence is the most straightforward approach to the solution of turbulent flows. In DNS, the governing equations are discretized and solved by accurate and higher order numerical schemes on extremely fine grids. The mesh should be sufficiently fine to resolve the smallest scales of motion to the order of Kolmogorov scale of motion. DNS can obtain an accurate three-dimensional, time-dependent solution of the governing equations completely free of modelling assumptions. DNS makes it possible to compute and visualize any quantity of interest, including some that are difficult to measure experimentally. It also gives spatial relationships between quantities of interest to obtain an insight into the detailed kinematics and dynamics of turbulent eddies. The very first DNS results were reported by Kim et al (1987). They performed DNS of a channel flow at $\operatorname{Re}_{\tau}=$ 180 and since then there have been many studies for different types of flows. A review article by Moin \& Mahesh (1998) gives up-to-date information, developments and detailed references till then. But, DNS has many limitations. To limit discretization errors in the solution, it is essential to use higher order numerical schemes. Such schemes are difficult to construct and they come with a large computational overhead. DNS calculation requires a large number of grid points to resolve the turbulence scales correctly. The number of grid points needed is proportional to the $9 / 4$ power of the Reynolds number, Re, defined by the velocity and length scale of the entire flow, and the cost of the computation is of the order of $\mathrm{Re}^{3}$. Because of these reasons, DNS has been limited to simple geometries and to low Reynolds numbers and its application to engineering-type problems may not be possible within the foreseeable future.

\section{Large Eddy Simulation (LES)}

Large Eddy Simulation (LES) is a technique that is a good compromise between the expensive DNS and less accurate RANS procedures. In LES, large eddies are resolved and the smallest, sub-grid-scale (SGS) eddies are modelled. This is achieved via spatially filtering the NavierStokes equations to obtain a set of equations that govern the resolved flow. LES is based on the underlying physics that the largest eddies, directly affected by the boundary conditions, carry most of the Reynolds stresses and must be resolved. The small-scale turbulence is weaker and thus contributing little to the change in Reynolds stresses. Also, small-scales are nearly isotropic and they have universal characteristics and thus amenable for modelling. Because the large-scale unsteady motions are represented explicitly, LES is expected to be more accurate and reliable than RANS for flows in which large-scale unsteadiness is significant - such as the flow over bluff bodies. Since LES involves modelling the smallest eddies, the smallest finite difference cells can be much larger than the Kolmogorov length scale, and much larger time steps can be used than that normally used in DNS calculations. Hence, for a given computing cost, it is possible to achieve much higher Reynolds numbers with LES than with DNS. 
Smagorinsky (1963) proposed the first model for the sub-grid-scale stresses based on gradient-diffusion concept for meteorological application. This subsequently gained popularity because of its simplicity and the Smagorinsky model is the most widely used model today. Deardorff (1970) was the first one who performed LES of channel flow at sufficiently high Reynolds number. Many newer models such as one equation model (Shumann, 1975; Yoshizawa \& Horiuti, 1985), scale similarity type models (Bardina et al 1980), dynamic model of eddy viscosity type (Germano et al 1991), mixed dynamic models (Zang et al 1993; Vreman et al 1994), one equation dynamic models (Ghosal et al 1995; Davidson, 1997) and dynamic two-parameter mixed model (Horitui, 1997; Morinishi \& Vasilyev, 2001) are then introduced. Study of SGS models to engineering and topographical problems has also been carried out (Vengadesan \& Nakayama, 2001; Nakayama \& Vengadesan, 2002).

\section{Limitations of LES}

Even though LES enjoyed a great deal of success (Moin, 2002), it suffers from limited validity all the way up to the solid boundary, especially at high Reynolds numbers. The near-wall region in high Reynolds number turbulent flow contains vortical structures, which play a key role. The presence of solid boundary affects the physics of the sub-grid scales in three ways. They are: (i) the growth of the small scales is inhibited by the presence of the wall; (ii) the exchange mechanisms between the resolved and unresolved scales are altered in the near-wall region. The sub-grid scales in this region may contain some significant Reynoldsstress producing events and (iii) the length scale of the energy-carrying large structures is Reynolds-number dependent near the walls.

A correct LES model must resolve all turbulent scales in the flow that contain most of the turbulent kinetic energy and Reynolds shear stress in a localized region. In such situations, the grid spacing $\Delta$ should be as minimum the local inertial length scale, $L=k^{3 / 2} / \varepsilon$, where $k$ is the turbulent kinetic energy and $\varepsilon$ is the turbulent dissipation rate. Near the walls, in boundary layers, the size of turbulent eddy scales are roughly proportional to the distance from the wall, which means that accurate LES model requires grid nearly as fine as those in DNS. Baggett (1997) estimated that the number of grid points necessary to resolve a channel flow properly with LES is approximately as $\operatorname{Re}_{\tau}^{2}$. Spalart et al (1997), reported that to perform LES for an airliner wing, with chord Reynolds number of about $10^{7}$, for example, one would require $10^{20}$ grid points. They also estimated that LES is unlikely to be used in aircraft design until the year 2045 despite the increase in computer speed. In wall-bounded flows, the instantaneous velocity on a solid wall is always zero and the no-slip boundary condition is the correct boundary condition for the time-averaged flow or the filtered flow. As explained above, if one does not resolve the near-wall accurately to capture the physics, the no-slip boundary condition is not appropriate. This is a serious limitation to perform LES for high Reynolds number practical flows.

Thus there is an urgent need to approximate the effects of near-wall structures on the larger scales through approximate boundary conditions without resolving the inner viscous regions. At the same time, these boundary conditions should be applicable to complex and separated flows, which exhibit different near-wall characteristics. Approximate boundary conditions or wall models should be devised in such a way to allow the use of LES grids on the outer flow scales and they should only be weakly dependent on the Reynolds number. LES performed this way is referred to as Very Large Eddy Simulation (VLES) (Speziale 1998). At present, this near-wall layer modelling is carried out by two different ways viz. (1) wall stress boundary condition; (2) hybrid strategy. The following sections explain these two strategies. 


\section{Wall stress models}

Wall stress models generally use information from outer flow near the walls to set the level of wall stress, by specifying a correlation between the velocity in the outer flow and stress at the wall. The wall stress is in turn fed back to the outer flow LES as an additional drag in the near-wall cells. Cabot \& Moin (2000), reviewed wall stress models developed. These approaches are similar to wall function methods employed in the high Reynolds number modelling using RANS simulations. Due to the fact that the wall functions are applied, the first point can be located in the logarithmic layer. Since the energy-producing vortical structures in the viscous and buffer regions do not have to be resolved, the wall function permits the use of coarser meshes near the walls. However, its applicability is questionable in separated flows and flow in complicated geometries. A new wall stress model with modification to loglaw incorporating spatial and temporal pressure gradients was attempted for topographical applications by Vengadesan \& Nakayama (2001).

\section{Hybrid models}

Spalart et al (1997) and Speziale (1998) proposed a new method in which non-slip conditions are still applied at the wall, and a hybrid type approach to turbulent flow simulation was attempted. Speziale (1998) proposed using Reynolds stress models in the near wall flow and a smoothing function to scale the eddy viscosity by a parameter $\alpha$ to match with that of outer flow obtained from LES. Speziale's idea was further promoted by Batten et al $(2003,2004)$ and referred to as Limited Numerical Scales (LNS) simulation. They used non-linear $k-\varepsilon$ RANS model near the walls. The latency factor $\alpha$ is expressed as a function of local turbulent length and velocity scales.

Spalart et al (1997) and Spalart (2000) used Spalart-Allmaras (SA) model (Spalart \& Allmaras, 1994) for constructing a hybrid type approach. The SA model, which is a one-equation RANS model, solves one transport equation to calculate eddy viscosity. The destruction term in the transport equation is inversely proportional to $d^{2}$ (length scale), where $d$ is the distance to the nearest wall. If $d$ is redefined suitably based on the local grid, then the eddy viscosity crosses over from the usual Spalart-Allmaras RANS eddy viscosity near the wall to proposed LES eddy viscosity, similar to that of Smagorinsky, away from the wall. Spalart (2000) calls this approach as 'Detached Eddy Simulation' (DES) since it is intended to be used in regions such as separated regions, in which only eddies that are detached from the surface must be resolved for accurate LES of the flow away from the walls. This DES model has a coefficient $C_{D E S}$, which is set to be 0.65 for homogeneous flows. DES technique has been demonstrated for flow over airfoil at different angle of attacks (Shur et al 1999), channel flow (Nikitin et al 2000), complex three-dimensional landing gear geometry flow (Stretlets 2001), for flow over Ahmed Car Reference model (Kapadia et al 2003) and for a flat plate at high angle of incidence (Breuer et al 2003).

Following Spalart's work, this hybrid approach is now being pursued by combining various other RANS models with SGS model and few such cases are given here. In general, if the same turbulence model is used to obtain both RANS eddy viscosity as well as SGS viscosity, it is referred to as DES and otherwise as Hybrid LES. Stretlets (2001) tried the philosophy of DES by choosing Mentor's (Mentor \& Rumsey, 1994) SST RANS model. Nichols \& Nelson (2003) proposed a multiscale $k-\varepsilon$ model based DES. Piomelli et al (2003) while simulating with SA model based DES, introduced a stochastic backscatter forcing at the 
interface to capture the correct level of turbulence. Davidson \& Peng (2003) made attempts by combining their own low Re $k-\omega$ model with one-equation SGS model of Yoshizawa (1993) and Fureby (1999) each one at a time. Tucker \& Davidson (2003) and Zhong \& Tucker (2004) have proposed combining Wolfshtein's (1969) $k-l$ RANS model with Yoshizawa (1993) and Fureby (1999)'s one equation SGS model. Abe (2005), and Temmerman et al (2005), have contemplated a hybrid model by combining non-linear $k-\varepsilon$ model with one-equation SGS model. In a parallel attempt, a Hybrid LES using a one-equation model for flow with massive separation was also carried out (Vengadesan \& Nakayama, 2005). Despite various proposals and attempts, the models are far from satisfactory if they had to be used to solve practical engineering problems. We believe that further research is essential in this area. Hence, in this report, some important issues in using hybrid models, treatment of interface zones and smoothing functions are discussed.

\subsection{Comments on hybrid models}

(i) Near the wall, solution by RANS is on a time-averaged framework even though unsteadiness is switched on during DES calculations. There is no clear evidence as to whether the modelling constants and closure approximation obtained based on time averaged flows are still valid in view of the fact that the SGS solution is obtained on spatially filtered framework away from the walls. The validity is questionable at the interface region in hybrid approaches, where both RANS and LES solution are expected to resolve the same range of turbulence scales.

(ii) Placing the interface between RANS and LES solution is a challenge. No universally acceptable approach is available. It is desirable to fix the interface independent of grid size and it should also be applicable to complex geometry and separated flows.

(iii) The type and amount of smoothing to ensure continuity of turbulent viscosity and to capture correct level of physical turbulence level at the interface is still a hot research topic.

Some of the popular DES methods are discussed in the following sub-sections.

\subsection{SA-based DES model}

The SA model is a one-equation turbulence model, solves the following transport equation for calculating eddy viscosity

$$
\begin{aligned}
\frac{D \bar{v}}{D t}= & C_{b 1}\left[1-f_{t 2}\right] \bar{S} \bar{v}+\frac{1}{\sigma}\left[\nabla \cdot((v+\bar{v}) \nabla \bar{v})+C_{b 2}(\nabla \bar{v})^{2}\right] \\
& -\left[C_{w 1} f_{w}-\frac{C_{b 1}}{\kappa^{2}} f_{t 2}\right]\left[\frac{\bar{v}}{d}\right]^{2}
\end{aligned}
$$

where $\bar{v}$ is the turbulent transport variable. The eddy viscosity $v_{\tau}$ is obtained as $v_{\tau}=\bar{v} f_{v 1}$. Definition of constants and functions used in this model can be found in Spalart et al (1997).

The destruction term in the transport equation is inversely proportional to $d^{2}$ (length scale), where $d$ is the distance to the nearest wall. If $d$ is replaced by $\widetilde{d}$ which in turn is defined suitably based on the local grid, then the eddy viscosity crosses over from the usual SpalartAllmaras RANS eddy viscosity near the wall to proposed LES eddy viscosity, similar to that 
of Smagorinsky, away from the wall. Hence, in this model, the interface location is dictated by the grid parameter through the switching condition

$$
\widetilde{d}=\min \left(d, C_{\mathrm{DES}} \max (\Delta x, \Delta y, \Delta z)\right)
$$

where $d$ is the wall normal distance, $\Delta x, \Delta y$ and $\Delta z$ are respectively grid width in $x-, y$ - and $z$-directions. The wall normal distance is compared against scaled LES characteristic filter width using the second term within the parenthesis of the above equation. Though it is very simple, this condition is undesirable, as it requires reasonable grid resolution in other two directions - stream-wise and span-wise in order to be realistic with streak length. It is also observed (Nitkin et al 2000) that when the interface is placed at viscous wall distance $\left(y u_{\tau} / v\right)$ of the order 20 (i.e. $y^{+}=O(20)$ ), resolution of scales is better and a smooth continuity of turbulent viscosity is ensured. When the interface is moved to $y^{+}=O(100-200)$ due to coarse wall-parallel grids, the turbulent eddy viscosity generated in the inner layer solution extends deep into the LES region, causing severe damping in the resolved motion and discontinuity in the velocity profile. Furthermore, very elongated unphysical very long streaks were found in the wall RANS region and skin-friction is under-predicted by about $15 \%$. To overcome this defect, Piomelli et al (2003) introduced stochastic backscatter forcing at a region below the interface. Although there is an improvement in the velocity distribution and skin friction prediction, it is not clear yet how to extend this method to practical problems.

\subsection{Multi-scale-based DES model (Nichols and Nelson, 2003)}

Here, the conventional two-equation model is split suitably to solve large scale and small scale of turbulent regions. The turbulent length scale $\left(L_{T}\right)$ defined in conventional way and grid length scale $\left(L_{G}\right)$, eddy viscosity and smoothing functions are given as:

$$
\begin{aligned}
L_{T} & =\max \left(6 \cdot 0 \sqrt{v_{t \mathrm{RANS}} / \omega, k_{\mathrm{RANS}}^{3 / 2} / \varepsilon_{\mathrm{RANS}}}\right) ; \quad L_{G}=\max (\Delta x, \Delta y, \Delta z) \\
v_{t} & =v_{t_{\mathrm{RANS}}} f_{d}+\left(1-f_{d}\right) v_{t_{\mathrm{LES}}} ; \quad v_{t \mathrm{LES}}=\min \left(0 \cdot 0845 L_{G} \sqrt{k_{\mathrm{LES}}}, v_{t \mathrm{RANS}}\right) \\
k_{\mathrm{LES}} & =k_{\mathrm{RANS}} f_{d} ;
\end{aligned}
$$

where

$$
f_{d}=\{1+\tanh [2 \pi(\Lambda-0 \cdot 5)]\} / 2 ; \quad \Lambda=1 \cdot 0 /\left[1 \cdot 0+\left(L_{T} / 2 L_{G}\right)^{4 / 3}\right]
$$

$v_{t \text { RANS }}$ and $\Omega$ are respectively unfiltered (large scale) eddy viscosity and mean flow vorticity, $k$ and $\varepsilon$ respectively are turbulent kinetic energy and dissipation rate and $\Lambda$ is the relative measure of the length scale. Unlike in original DES, where the transition is effected only based on grid information, here transition is designed based on local grid size and turbulent length scale. This is expected to provide smooth variation of eddy viscosity, reduced mismatch of velocity profile and minimum non-physical turbulence physics at the interface. The applicability of this method to three-dimensional problems and its sensitivity to mesh refinement and time step are not known.

\subsection{Zhong and Tucker model (2004)}

Here, a transitional zone (subscript TRAN) as shown in the figure 1 is introduced. In this zone, expression for length scale and modelling constants are evolved as

$$
l_{\mathrm{TRAN}}=(1-s) l_{\mathrm{RANS}}+s l_{\mathrm{LES}} ; \quad c_{\mathrm{TRAN}}=(1-s) c_{\mathrm{RANS}}+s c_{\mathrm{LES}}
$$




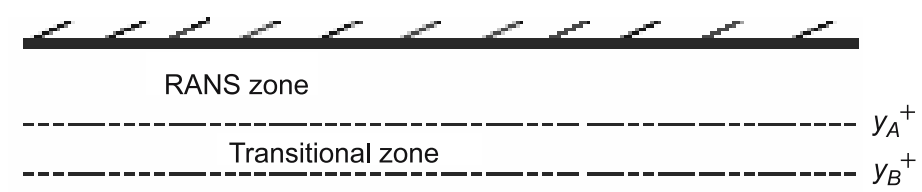

LES zone

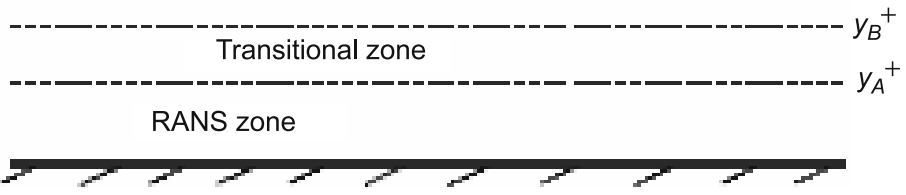

Figure 1. RANS/LES interface proposal (figure 1 of Zhong \& Tucker, 2004).

where, $c$ represents modelling constant. The parameter $s$ is a weighting function, and a value of 0 or 1 represents RANS or LES zone respectively. Different weighting functions like linear, power and harmonic were attempted by the proponents. To correctly capture near wall turbulence level, under-relaxation procedure and larger near wall time steps were also attempted by Zhong \& Tucker (2004). The proposed model is calibrated for channel flow and applied to heat transfer problems. Although there are improvements, the proposed velocity under-relaxation and time step filtering was found to give less convincing results. However, when applied to convection heat transfer over a matrix of surface mounted cubes the predicted heat transfer results from the LES and hybrid LES seem to be quite close.

\subsection{Two-layer hybrid model (Temmerman et al 2005)}

In this model, the two-equation, non-linear $k-\varepsilon$ model of Abe et al (1994) was used near the walls. The RANS model is merged with the one-equation SGS model of Yoshizawa \& Horiuti (1985) to obtain a hybrid model. At the interface, velocities, modelled turbulence energy and turbulence viscosity are interchanged between two zones. One of the key suggestions proposed by Temmerman et al (2005) is the continuity constraint of the turbulent viscosity across the interface via the relation,

$$
v_{\mathrm{LES}}^{\mathrm{mod}}+v_{\mathrm{LES}}^{\mathrm{res}}=v_{\mathrm{RANS}}^{\mathrm{mod}}+v_{\mathrm{RANS}}^{\mathrm{res}}
$$

The modelled and resolved part of LES viscosities are obtained in usual manner. Following this constraint, the coefficient $C_{\mu}$ in the RANS model is forced to take an interface value consistent with the condition:

$$
C_{\mu, \text { int }}^{\mathrm{av}}=\frac{\left\langle v_{\mathrm{LES}}^{\mathrm{mod}}\right\rangle}{\left\langle f_{\mu} k^{2} / \varepsilon\right\rangle}
$$

In the above expression, the angular bracket means averaging over homogeneous directions, $f_{\mu}$ is a damping function defined as part of RANS model and $k$ and $\varepsilon$ are respectively turbulent kinetic energy and dissipation rate. The boundary condition for $k$ at the interface is supplied by LES model and for $\varepsilon$ is evaluated from empirical relation $-k^{3 / 2} / C_{l} y$, where $y$ is the wall normal distance. Disadvantages of this model are (i) supply of sub-grid-scale energy from the LES region at the interface and (ii) difficulty in the definition of characteristic wall distance 


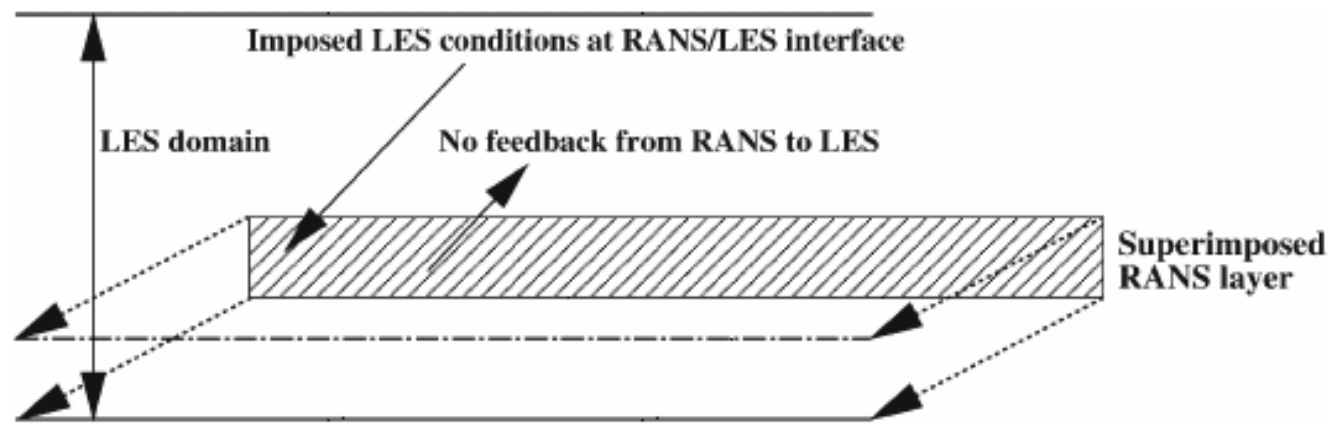

Figure 2. RANS/LES interface proposal (figure 3 of Temmerman et al 2005).

(y) for complex flows. The constant evaluated at the interface is used to effect the smooth transition from the RANS value of $C_{\mu}=0.09$ to LES value away from the walls.

$$
C_{\mu}=0.09+\left(C_{\mu, \text { int }}-0.09\right) \frac{1-\exp (-y / \Delta)}{1-\exp \left(-y_{\text {int }} / \Delta_{\text {int }}\right)}
$$

The manner in which the tests have been conducted is given schematically in figure 2 and the proposed model is tested initially for a channel flow at $\operatorname{Re}_{\tau}=590$ and 2000.

At the lower Reynolds number, the interface was placed at $y^{+}=65$, while for higher Reynolds number it was placed at two different locations, $y^{+}=48$ and 135 . In both cases, log-law in the velocity profile was reproduced accurately. The variations of resolved and modelled parts of shear stress and turbulence-energy distributions were consistently better than RANS models. However, solutions are found to be sensitive to interface location for a critical range of $y^{+}$within which the interface exists. The proposed model is applied further to separated flows at low a Reynolds number within a channel, one wall of which forms hillshaped periodic constriction. For this test case the results were inconclusive.

\subsection{Abe model (2005)}

In order to resolve near-wall anisotropy better, Abe (2005) proposed using a non-linear twoequation model in the RANS region. Non-linear RANS models are also gaining popularity especially for flows with curved streamlines. However, RANS calculations with non-linear models demand higher computational resources as they involve calculation of higher order of velocity terms in comparison with their linear counterparts.

Different length scale definitions as given below were used.

$$
\begin{aligned}
\Delta & =(\Delta x * \Delta y * \Delta z)^{1 / 3} ; \quad \Delta=\max (\Delta x, \Delta y, \Delta z) ; \\
\Delta & =\sqrt{\Delta x^{2}+\Delta y^{2}+\Delta z^{2}} ; \quad \Delta=\sqrt{\max (\Delta x \Delta y, \Delta y \Delta z, \Delta z \Delta x)} \\
& =\sqrt{\text { the max. area among the cell faces }}
\end{aligned}
$$

The last expression expected to work even for non-orthogonal or the unstructured grid systems.

Smoothing function used is given as

$$
\tau_{i j}=\left(1-f_{h b}\right){\overline{u_{i} u_{j}}}_{(\mathrm{RANS})}+f_{h b} \tau_{i j(\mathrm{LES})} ; \quad f_{h b}=1-\exp \left\{-\left(\frac{n}{C_{h b} \Delta}\right)^{6}\right\}
$$


After testing various values, the recommended value for the hybrid constant is $C_{h b}=4 \cdot 0$. Only channel flow was attempted. Even though smooth transition and matching of velocity profile is observed, results for turbulent stresses and turbulent kinetic energy was not appealing. At higher Reynolds number, the results seem to be sensitive to grid resolution. It was also observed that this model over predicts skin friction.

\section{Conclusions}

It appears that the hybrid LES approach has promising features and it has the potential to solve large scale engineering problems of practical interest on realistic meshes. Although predictions for the simple channel flow results are only marginally better than RANS calculations, its relatively easy application to complex problems is encouraging. Two-equation based RANS models near the wall are emerging as better choice over the one-equation models. The solution seems to improve if one uses low-Reynolds number version or non-linear version of RANS models. Nevertheless, such models impose further constraint on the placement of the first point from the wall and thus affect the computational cost, often increasing it significantly. Appropriate condition at the interface for the variables used in the transport equations of RANS model needs careful calculation from the available LES data. Not only smoothing of eddy viscosity, but also the modelling constants across the transition zone with the continuity constraint at the interface location is also required. Explicit specification of interface location is obviously not a desirable one. The location should be automatically decided based on the local grid information without imposing further limitation on grid in other directions. Finally, application of smoothing function, filter and length scale definition to unstructured mesh environment needs further research.

This report was prepared while the first author was in University of Wales Swansea, Swansea, UK. The financial support from Department of Science and Technology (DST), India and Royal Society, UK is gratefully acknowledged.

\section{References}

Abe K 2005 A hybrid LES/RANS approach using an anisotropy-resolving algebraic turbulence model. Inter. J. Heat and Fluid Flow 26: 204-222

Abe K, Kondoh T, Nagano Y 1994 A new turbulence model for predicting fluid flow and heat transfer in separating and re-attaching flows - I, Flow field calculations. Int. J. Heat and Mass Transfer 37: 139-151

Baggett J S 1997 Some modelling requirements for wall models in large eddy simulation. Ann. Res. Briefs, NASA Ames/Stanford Center for Turbulence Research 123-134

Bardina J, Ferziger J H, Reynolds W C 1980 Improved sub-grid-scale models for large eddy simulation. AIAA paper $80-1357$

Batten P, Goldberg U C, Chakravarthy S R 2003 Using synthetic turbulence to interface RANS and LES. AIAA Paper 2003-0427

Batten P, Goldberg U C, Chakravarthy S R 2004 Interfacing statistical turbulence closures with largeeddy simulation. AIAA, J. 42(3): 485-492

Breuer M, Jovicic N, Mazaev K 2003 Comparison of DES, RANS and LES for the separated flow around a flat plate at high incidence. Inter. J. Numer. Meth. Fluids 41: 357-388 
Cabot W, Moin P 2000 Approximate Wall boundary conditions in the large-eddy simulation of high Reynolds number flows. Flow, Turbul. Comb. 63: 269

Davidson L 1997 Large Eddy Simulation: A dynamic one-equation sub-grid model for threedimensional recirculating flow. 11th Inter. Symp. Turbulent Shear Flow, Greenbole 3: 26.1-26.6

Davidson L, Peng S H 2003 Hybrid LES-RANS modelling: A one-equation SGS model combined with a $k-\omega$ model for predicting recirculating flows. Inter. J. Numer. Meth. Fluids 43: $1003-1018$

Deardorff J W 1970 A numerical study of three-dimensional turbulent channel flow at large Reynolds numbers. J. Fluid Mech. 41(2): 453-480

Fureby C 1999 Large eddy simulation of rearward-facing step flow. AIAA J. 37: 1401-1411

Germano M, Piomelli U, Moin P, Cabot W H 1991 A dynamic sub-grid scale eddy viscosity model. Phys. Fluids A 3: 1760-1765

Ghosal S, Lund T, Moin P, Akselvoll K 1995 A dynamic localization model for large eddy simulation of turbulent flows. J. Fluid Mech. 286: 229-255

Horiuti K 1997 A new dynamic two-parameter mixed model for large-eddy simulation. Phys. Fluids 9: 3443-3464

Johnson R W (Ed.) 1998 Turbulence modelling and simulation, The Handbook of Fluid Dynamics, (CRC: Spring-Verlag)

Kapadia S, Roy S, Wurtzler K 2003 Detached eddy simulation over a Reference Ahmed Car model, 41st Aerospace Science Meetings and Exhibit, AIAA 2003-0083

Kim J, Moin P, Moser R 1987 Turbulence statistics in fully developed channel flow at low Reynolds number. J. Fluid Mech. 177: 133-166

Mentor F R, Rumsey C L 1994 Assessment of two-equation turbulence models for transonic flows. AIAA 94-2343

Moin P 2002 Advances in large eddy simulation methodology for complex flows. Inter. J. Heat and Fluid Flow 23: 710-720

Moin P, Mahesh K 1998 Direct numerical simulation - A tool in turbulence research. Ann. Rev. Fluid Mech. 30: 539-578

Morinishi Y, Vasilyev O V 2001 A recommended modification to the dynamic two-parameter mixed sub-grid scale model for large eddy simulation of wall bounded turbulent flow. Phys. Fluids A 13: 3400-3410

Nakayama A, Vengadesan S N 2002 On the influence of numerical schemes and sub-grid-stress models on large eddy simulation of turbulent flow past a square cylinder. Inter. J. Numer. Meth. Fluids 38: 227-253

Nichols R H, Nelson C C 2003 Application of hybrid RANS/LES turbulence models, 41st Aerospace Science Meetings and Exhibit, AIAA 2003-0083

Nikitin N V, Nicoud F, Wasistho B, Squires K D, Spalart P R 2000 An approach to wall modelling in large-eddy simulations. Physics of Fluids 12(7): 1629-1632

Piomelli U, Balaras E, Pasinato H, Squires K D, Spalart P R 2003 The inner-outer layer interface in large eddy simulations with wall-layer models. Int. J. Heat and Fluid Flow 24: 538-550

Peyret R, Krause E 2000 Advanced Turbulent flow Computations, CISM courses and lectures, No. 395, (New York: Springer Wein)

Pope S B 2000 Turbulent flows, (New York: Cambridge University Press)

Shumann U 1975 Sub-grid scale model for finite difference simulations of turbulent flows in plane channels and annuli. J. Comput. Phys. 18: 376-404

Shur M, Spalart P R, Strelets M, Travin A 1999 Detached-eddy simulation of an airfoil at high angle of attack, Engineering Turbulence Modelling and Experiments - 4, (Eds) W Rodi and D Laurence, 669-677

Smagorinsky J 1963 General circulation experiments with primitive equations-I, The basic experiment. Mon. Weather Review 91: 99-165

Spalart P R, Allmaras S R 1994 One equation turbulence model for aerodynamics flows. La Rech. Aerospatiale. 1: 5-21 
Spalart P R 2000 Strategies for turbulence modelling and simulations. Int. J. Heat and Fluid Flow 21: 252-263

Spalart P R, Jou W H, Strelets M, Allmaras S R 1997 Comments on the feasibility of LES for wings, and on a Hybrid RANS/LES approach, Advances in DNS/LES, Proc. 1st AFOSR Inter. Conf. on DNS and LES, (NY: Greyden Press)

Speziale C G 1998 Turbulence modelling for time-dependent RANS and VLES: A review. AIAA. J. 36: $173-184$

Stretlets M 2001 Detached eddy simulation of massively separated flows. 39th Aerospace Sciences Meeting and Exhibit, AIAA Paper 2001-0879

Temmerman L, Hadziabdic M, Leschziner M A, Hanjalic K 2005 A hybrid two-layer URANS-LES approach for large eddy simulation at high Reynolds numbers. Inter. J. Heat and Fluid Flow 26: $173-190$

Tucker P G, Davidson L 2003 Zonal $k-l$ based large eddy simulations. Comput. \& Fluids 33: 267-287

Vengadesan S N, Nakayama A 2001 An investigation of appropriate LES method for wind flow over two-dimensional topography. Ann. J. Hydraulic Eng. JSCE 45: 223-228

Vengadesan S, Nakayama A 2005 Evaluation of LES models for flow over bluff body from engineering application perspective. Sädhanā 30: 11-20

Vreman B, Geurts B, Kuerten K 1994 On the formulation of the dynamic mixed sub-grid model. Phys. Fluids A 6: 4057-4059

Wilcox D C 2006 Turbulence modelling for CFD, 3rd ed. (LA, USA: DCW industries)

Wolfshtein M 1969 The velocity and temperature distribution in one-dimensional flow with turbulence augmentation and pressure gradient. Int. J. Heat and Mass Transf. 12: 301-318

Yoshizawa A, Horiuti K 1985 A Statistically-derived sub-grid-scale kinetic energy model for the large-eddy simulation of turbulent flows. J. Phys. Soc. Japan 54: 2834-2839

Yoshizawa A 1993 Bridging between eddy-viscosity-type and second order models using a two-scale DIA. 9th Inter. Sympo. on Turbulent Shear Flow 23.1.1-23.1.6

Zang Y, Street R L, Koseff J R 1993 A dynamic mixed sub-grid-scale model and its application to turbulent recirculating flows. Phys. Fluids, A 3186-3196

Zhong B, Tucker P G $2004 k-l$ based hybrid LES/RANS approach and its application to heat transfer simulation. Inter. J. Numerical Methods in Fluids 46: 983-1005 